\title{
Estudio comparativo sobre la comprensión de textos de futuros docentes
}

\author{
(Comparative study related to texts comprehension of upcoming \\ professors)
}

\author{
Mirián Adriana Noriega Jacob iD , Centro Regional de Formación Docente de Estado de \\ Sonora, Sonora, México
}

\author{
Volumen 4, Número 1 \\ Enero-Junio de 2019 \\ p. $1-25$
}

Este número se publicó el 30 de junio de 2019

Artículo recibido: 11 de mayo de 2018

Artículo aprobado: 3 de abril de 2019

ISSN: 2448-5942, doi: https://doi.org/10.36799/el.v4i1.78

Citar este artículo como:

Noriega Jacob, M. A. (2019). Estudio comparativo sobre la comprensión de textos de futuros docentes. Estudios גambda. Teoría y práctica de la didáctica en Lengua y Literatura. , 4(1), 1-25. https:// doi.org/10.36799/el.v4i1.78

Derechos de autor: El autor o autores conservan en todo momento sus derechos morales y patrimoniales sobre la obra; la obra no se puede alterar, transformar o ampliar; siempre debe reconocerse la autoría del documento referido. Ninguna de

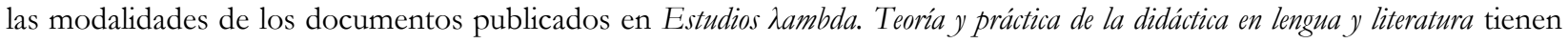
fines comerciales de naturaleza alguna.

Los contenidos de este artículo están bajo una licencia de Creative Commons Atribución No Comercial- Sin Derivadas 4.0 Internacional (c) $(7) \Theta$ 


\title{
Estudio comparativo sobre la comprensión de textos de futuros docentes
}

\author{
Comparative study related to texts comprehension of upcoming professors
}

\author{
Mirián AdRIANA NORIEGA JACOB ${ }^{1}$
}

\begin{abstract}
RESUMEN
El objetivo principal de este trabajo es comparar la comprensión de textos de estudiantes de primer y séptimo semestre de tres escuelas normales del Estado de Sonora. El instrumento utilizado fue diseñado para participantes estudiantes sonorenses de educación superior, el cual incluye una lectura de interés público y 24 ítems con cuatro posibles respuestas; los reactivos permiten explorar tres niveles de comprensión: 1) extracción u obtención de información, 2) interpretación de textos y 3) razonamiento de contenido. El número total de participantes del estudio es 303 normalistas, 107 inscritos en primer semestre y 196 de séptimo semestre. En general, se observa que los participantes del estudio de primer y de séptimo semestre se ubican en un rango medio de comprensión de textos. Asimismo, se advierte sobre la inexistencia de diferencia significativa entre los estudiantes de primer y séptimo semestre en los resultados obtenidos en función a la comprensión de textos. A partir de lo anterior se señala la importancia de crear espacios que promuevan las competencias lectoras de los estudiantes normalistas, pues ellos en gran medida realizarán sus prácticas docentes a partir de sus propias competencias y experiencias lectoras.
\end{abstract}

PALABRAS CLAVE: comprensión, lectura, estudiantes.

\section{ABSTRACT}

The main purpose of this work is to compare the reading comprehension of students from first and seventh semester among three superior schools of the state of Sonora. The instrument utilized was designed for contestants for sonoran context of high education, which includes a lecture of public interest and 24 items with four possible answers; the choices allow to explore three levels of comprehension: 1) extraction of information, 2) texts understanding and 3) content argumentation. The total number of contestants from the study is 303 normalists, 107 signed up on first semester and 196 from seventh semester. Overall, it is noted that the contestants of the study from first and seventh semester are placed in a middle range of reading comprehension. Additionally, it is warned about the absence of a meaningful difference among the students from first and seventh semester in the results obtained according to texts comprehension. Based on the previous facts, it is emphasized the importance to create opportunitied that promote reading competitions among normalist students, since they will carry out their teaching practices based on their own competences and reading experiences.

KEY WORDS: comprehension, reading, students.

\footnotetext{
${ }^{1}$ Mirián Adriana Noriega Jacob es Doctora en Humanidades por la Universidad de Sonora; actualmente es investigadora en CRESON. Sus líneas de investigación son: procesos de enseñanzas y aprendizajes de la lengua, didáctica de la lengua, comprensión de textos. Su publicación reciente es: Noriega Jacob, Mirián Adriana. “¿Para qué enseñar español en educación primaria?”. Estudios Lambda. Teoría y práctica de la didáctica de la lengua y literatura, 2 (2017): 72-93. Correo electrónico: m.noriega@creson.edu.mx,noriega_adriana@hotmail.com. ORCID:

https://orcid.org/0000-0002-2968-8422
}

Artículo recibido: 11 de mayo de 2018

Artículo aprobado: 3 de abril de 2019

Noriega doi: https://doi.org/10.36799/el.v4i1.78Volumen 4, Número 1, Año 2019, ISSN: 2448-5942 


\section{INTRODUCCIÓN}

Las prácticas de lectura y la comprensión de textos en México han ocupado lugares importantes en el desarrollo de investigaciones y de programas para el fomento de lectura en la población, ya que a partir de la última década del siglo XX se realizaron estudios internacionales donde se mostró la escasa práctica lectora de los mexicanos y su correlación con la comprensión de textos.

A partir de los resultados de la Prueba PISA y de evaluaciones nacionales donde se muestran los bajos logros en las competencias lectoras de los estudiantes de Educación Básica y Media Superior, se han diseñado e implementado diversas estrategias para promover la práctica y la comprensión de lectura en contextos áulicos y sociales. En el caso de las acciones educativas se han concentrado la atención en el estudiante de Educación Básica y Media Superior, dejando de lado las prácticas y la comprensión de textos de las personas que llevan a cabo las acciones de promoción de lectura y de estrategias que promuevan la comprensión de textos. El profesor de los distintos niveles educativos es una figura que influye en el proceso de aprendizaje y de experiencias lectoras que viven los estudiantes, ya que el docente través de su experiencia lectora y didáctica crea espacios de interacción y ambientes de lectura para la formación de lectores.

Este documento compara resultados obtenidos al explorar la comprensión de textos de estudiantes inscritos en primer y séptimo semestre de tres escuelas normales del Estado de Sonora. Este texto incluye algunas cuestiones teóricas relacionadas con los modelos de lectura como base al estudio de las competencias lectoras, presenta el método utilizado para la exploración de la comprensión de textos de los participantes, posteriormente comparte los resultados obtenidos y finalmente se enuncian algunas reflexiones sobre los datos.

\section{MODELO DE LECTURA: ASCENDENTE}

La concepción tradicional del procesamiento de la lectura se basa en la información que se propaga de 'abajo-arriba' a través del sistema, desde el reconocimiento visual de las letras hasta el procesamiento semántico del texto como un todo, sin que la relación inversa sea necesaria (Alonso y Mateos, 1985). De acuerdo con Seoane (2002), el modelo ascendente tiene dos pasos. El primero es el reconocimiento visual de las palabras y la comprensión oral, y el segundo es el análisis de la información presente. Esto ha llevado a que el modelo se caracterice por ser 
unidireccional partiendo de la información planteada y leída de un texto, la cual desencadena el procesamiento de abajo (visual) hacia arriba (semántico) (Bruning et al., 2007; Galera, 2003; González Casaravilla, 2013).

Solé (2004) y Aguirre et al. (2002) afirman que el procesamiento de la lectura según el modelo ascendente inicia con el reconocimiento de las grafías, después los sonidos, las palabras, frases, oraciones hasta lograr el significado. Esto permite suponer que el lector es capaz de comprender un texto, si puede extraer la información. Sin embargo, el hecho de ser un modelo unidireccional y lineal dificulta la comprensión del lector, ya que el manejo deficiente de las pequeñas unidades de análisis representa un obstáculo para captar el mensaje totalmente.

El rol del lector en este modelo de lectura es de receptor, porque la información que recibe son solo datos aislados (Viglione, López y Zabala: 2005); lo cual hace que el lector al procesar la información no sea realmente con base en el significado porque deja de lado aspectos de la historia académica, personal y profesional del sujeto. Incluso, el modelo ascendente limita a la lectura literal, de tal modo que la percepción de los estímulos visuales que aparecen sobre la página, culmina con la reconstrucción en la mente del lector del significado que el autor de un texto pretende transmitir (Rivera, Forteza y Rivera: 2006).

Durante varios años la enseñanza de la lectura se concibe como una graduación de contenidos. Los adultos, quienes con base en su experiencia con el uso de la lengua escrita, determinan qué es lo más sencillo o complicado para los nuevos alfabetos. Según Viglione, López y Zabala (2005), lo anterior provoca una división artificial entre la lectura y la comprensión, pues se parte de la idea que una vez que se lee se podrá comprender un texto.

\section{MODELO DE LECTURA: DESCENDENTE}

El modelo descendente se caracteriza por ser un proceso de comprensión lectora determinado por las expectativas y/o conocimientos previos que posee el lector en relación con los temas abordados en el texto. De modo tal, que "comprender consiste en seleccionar los esquemas existentes" (Seoane, 2002). En esta selección, el cerebro del ser humano recuerda información específica cuando surgen excepciones a nuestras reglas o resúmenes, o bien, cuando se adquiere alguna significación emotiva en alguna situación particular (Smith, 1997). Es decir, el cerebro es capaz de dar sentido al mundo a partir de lo que ya conoce, aquel elemento o 
situación que no se puede relacionar con lo conocido no tiene sentido para el individuo (Bruning et al., 2007; Galera, 2003; Goodman, 1967; González Casaravilla, 2013).

El juego de la predicción en la lectura dentro del modelo descendente es valioso, puesto que le permite al sujeto descartar anticipadamente aquellas alternativas improbables de la información planteada en el texto. Asimismo, Aguirre et al. (2002) afirman que el significado de una lectura inicia con las predicciones que realiza el lector y progresivamente analiza aquellas unidades que son difíciles para su comprensión. En otras palabras, el lector no procede letra por letra, sino el lector utiliza sus conocimientos y sus recursos cognitivos para encontrar y anticiparse al contenido que ofrece el texto; así, poco a poco verifica la información (Rivera et al.: 2006). De acuerdo con Solé (2004), a mayor información que posea un lector acerca de un texto, ya sea del tema, del léxico, de las estrategias discursivas, etc., menos necesitará prestar atención a las unidades necesarias para construir su propia interpretación. El proceso de la lectura descendente es secuencial y jerárquico, a partir de las ideas previas y anticipaciones que se formula el lector que, a su vez va comprobando, pero no de las unidades gráficas menores.

Las bases teóricas del modelo descendente planteadas por Goodman (2006) señalan, por un lado, que el lector busca el significado del texto, no el reconocimiento de las palabras. El autor fundamenta dicha idea en que los ojos son herramientas del cerebro, éstos dicen dónde ver y qué buscar. Por otro lado, el lenguaje funciona mientras leemos y es a través del uso del lenguaje como encontramos el significado de la lectura. $\mathrm{O}$ sea, el sentido y el sinsentido en cualquier idioma radican en el acervo de experiencia del lector y su familiaridad con términos y conceptos que encuentra. Así, cuando el lector trata de entender un texto que incluye conceptos, ideas y terminología que no le son familiares, solo puede concebirlo como sin sentido. Si lee en vOZ alta, puede producir pronunciaciones y entonaciones bastantes aceptables, de manera que suenen como un lenguaje que tenga sentido para el lector.

La principal característica del modelo descendente es la amplia confianza que le otorga al lector respecto a su conocimiento semántico y sintáctico; de esta manera, se minimiza la información gráfica. Además, autores como Alonso y Mateos (1985), mencionan que los buenos lectores, al interpretar el significado del texto, se sirven más de sus conocimientos sintácticos y semánticos de forma anticipada que de los detalles gráficos. Sin embargo, la desventaja más fuerte de esta propuesta es el gran énfasis en que la predicción minimiza los detalles, lo cual es altamente problemático cuando el conocimiento sintáctico y/o semántico de la lengua no es Noriega doi: https://doi.org/10.36799/el.v4i1.78Volumen 4, Número 1, Año 2019, ISSN: 2448-5942 
suficiente, de modo tal que el lector tiene que recurrir a su información léxica y ortográfica (Aguirre et al., 2002).

La propuesta de Goodman es una de las más destacadas en relación a los modelos descendentes (top down). Goodman, al investigar la lectura de niños, se percata de que el sujeto se anticipa al contenido de un texto y que su lectura es utilizada para confirmar la información. Para este autor la lectura es concebida como un “juego psicológico de adivinanzas”, además, ésta se lleva a cabo en cuatro ciclos: 1) estímulo visual, 2) identificación de letras y palabras, 3) identificación de estructura del texto, y 4) construcción del significado (Bruning et al., 2007; González Casaravilla, 2013).

\section{MODELO DE LECTURA: INTERACTIVO}

El modelo interactivo implica una participación recíproca por parte del lector y del autor. En este modelo el lector debe desarrollar habilidades que suponen el modelo ascendente y el modelo descendente. El modelo interactivo es bidireccional, ya que el lector procesa los esquemas en ambas direcciones. Pero si alguno de los procesos no se ejecuta eficazmente, es muy probable que surjan problemas en la comprensión lectora (Viglione et al., 2005).

El procesamiento bidireccional (texto-conocimiento) facilita la asimilación de la información de orden inferior consistente con las expectativas del lector; además, el sujeto es consciente de aquella información que no se asemeja con las hipótesis previamente formuladas (Alonso y Mateo, 1985).

El significado no reside en las palabras, las frases, los párrafos, ni en el texto considerado globalmente, sino se encuentra en el lector, que activamente construye o representa la información del texto acomodándola a su conocimiento sobre el mundo y sus propósitos de comprensión en un momento dado. De este modo, la construcción del significado es el resultado de la interacción entre el texto, el contexto y los esquemas de conocimiento lingüístico, situacional, actitudinal, requerimientos de la tarea, etc. (Alonso y Mateo, 1985).

Asimismo, en el modelo interactivo se conjugan el modelo psicolingüístico y la teoría del esquema (Monroy et al., 2009). El modelo psicolingǘstico considera que leer no solamente es descodificar el significado expuesto y explícito, sino también es necesario recuperar y probar 
aquellas inferencias que se han elaborado y cubrir los espacios semánticos que propone el texto leído (Cassany, 2008).

La teoría del esquema plantea que los esquemas no únicamente almacenan el conocimiento, sino además almacenan la información necesaria para utilizarlo. Estos esquemas se caracterizan por tener variables vinculadas a diferentes aspectos del ambiente en diferentes evocaciones de los esquemas, es decir, los elementos tienen tintes sintácticos porque su función es identificar los distintos aspectos de la situación con las variables omitidas y restringir las operaciones como valores omitidos para variables que no han sido observadas. Los esquemas se caracterizan por estar relacionados con una red o árbol de subesquemas, es decir, redes semánticas (Viglione et al., 2005).

Por otra parte, Solé (2004) menciona que cuando el lector se sitúa ante el texto, los elementos que lo componen provocan expectativas a distintos niveles lingüísticos, por ejemplo gráfico-léxico, y a medida que procesa la información en cada uno de ellos funciona como input para el próximo nivel, en este momento el procesamiento es ascendente. Pero simultáneamente, el texto genera también expectativas semánticas de su significado global. Estas guían la lectura y buscan su verificación en indicadores de nivel inferior (léxico, sintáctico, grafo-fónico) a través de su proceso descendente. Es por esto que autores como Monroy et al. (2009) y Viglione et al. (2005) describen al modelo interactivo como bidireccional, porque el lector utiliza simultáneamente su conocimiento del mundo y del texto para construir una interpretación.

De acuerdo con Aguirre et al. (2002), toda lectura implica varios procesos. De manera que si un lector no es eficiente en una estrategia, se puede apoyar en otros procesos para compensar. En el caso de los buenos lectores, con su experiencia lectora han desarrollado numerosas y diversas estrategias de lectura que pueden adaptar a los distintos textos que utilizan. Las habilidades y las estrategias que se utilizan en la lectura, según el modelo interactivo, permiten la interacción durante el procesamiento y la interpretación del texto.

A partir de lo anterior, la comprensión lectora en el modelo interactivo puede ser entendida como un proceso flexible, que se adapta a los propósitos de lectura en cada momento. Para lograr la comprensión es fundamental que el sujeto considere simultáneamente el conocimiento previo y elementos textuales como sus características y contenido. Sin embargo, cuando la comprensión no se logra puede atribuirse a varias razones. Por un lado, la ausencia de 
esquemas pertinentes por parte del lector; en caso de que sí se posea los esquemas apropiados, es necesario que el sujeto cuente con claves en el texto para sugerirlos. Por otro lado, es posible que el lector interprete el texto de una manera distinta sin coincidir con la que el autor desea transmitir (Alonso y Mateos, 1985; Monroy et al., 2009; Rivera et al., 2006; Viglione et al., 2005).

Por otra parte, Seoane (2002) señala que desde la perspectiva de este modelo de lectura, es importante: el establecimiento de un propósito de lectura; el uso de claves del texto y del conocimiento previo para generar inferencias y predicciones sobre el significado; y la implementación de estrategias de control, como búsqueda del conocimiento propio; así la aplicación de estrategias supervisan la regulación de la comprensión.

El modelo de Kinstch y van Dijk (1983) es una propuesta teórica interdisciplinar, ya que retoma para su elaboración los elementos lingüísticos, el procesamiento de la información y propuestas del constructivismo cognitivo. De esta manera, el modelo adjudica el proceso a multiplicidad de procesos que se realizan en paralelo y secuenciales. El modelo supone una lectura superficial y profunda del texto. Para la primera se plantea una correspondencia de elementos lingüísticos y en la lectura profunda se procesa la información en dos niveles: texto base y modelo de situación (González Casaravilla, 2013).

\section{MODELO DE LECTURA: TRANSACCIONAL}

El modelo transaccional supone que el texto no es un objeto, sino un potencial actualizado durante el acto de lectura. Además, el texto es un sistema abierto y por lo tanto la variación en la respuesta interpretación es esperada. Así, la comprensión surge de la compenetración lectortexto, es decir, el lector y el texto son dependientes en el proceso, en el cual cada elemento es visto como los aspectos o fases de una situación total (Dubois, 1989; Viglione et al., 2005). De acuerdo con Dubois (1989), la lectura en esta perspectiva, al igual que la escritura, es un evento realizado en momentos particulares bajo las intenciones específicas influidas por factores internos o externos, en el cual se congregan el lector y el texto en condiciones particulares.

En una primera aproximación, el modelo transaccional pudiera confundirse con el modelo interactivo. Sin embargo, la diferencia en el modelo transaccional es que los elementos independientes no son significativos (Monroy et al., 2009). 
En el proceso de comprensión, el lector presta atención a elementos informativos del texto de una manera selectiva adoptando una postura que puede ser consciente o inconsciente; dichas posturas son la estética y la eferente. En la primera el sujeto permanece concentrado en lo que piensa y siente durante el acto de lectura; es decir, se caracteriza por aspectos afectivos, emotivos y cualitativos. En la segunda, la postura eferente, el lector se concentra en el significado aparentemente lógico, verdadero y en aspectos cognitivos (Aguirre et al., 2002; Dubois, 1989).

Es decir, el lector selecciona del texto solo la información más relevante de acuerdo a sus conocimientos, experiencia de vida y objetivo de lectura. El texto construido por el lector será diferente al escrito por el autor, ya que las inferencias y referencias se basan en esquemas personales de cada uno y en el almacén de conocimientos, pensamientos y sentimientos. De este modo, la interacción no es solo entre el lector y el texto sino también con los culturales, sociales y personales, lo cual abre la posibilidad de que el significado del texto y el que surge o se construye a partir de la lectura no son nunca idénticos sino aproximados, esto último es resultado de los factores sociales y personales que entran en la situación de lectura para condicionar la selección de la atención (Dubois, 1989; Viglione et al., 2005).

\section{MÉTODO}

Los datos que se presentan en este documento se obtuvieron de 303 estudiantes de tres escuelas normales del Estado de Sonora, 107 de ellos son de primer semestre y 196 de séptimo semestre (véase tabla 1). El 99.97\% del grupo de participantes son estudiantes regulares, asimismo, el $77.55 \%$ son mujeres (véase tabla 2). La metodología empleada para este estudio se caracteriza por ser cuantitativa y de corte no probabilístico, ya que la participación de los alumnos fue a partir del total que se encontraban presentes el día que se aplicó el instrumento.

\begin{tabular}{|l|l|l|l|}
\hline & I Semestre & VII Semestre & Total \\
\hline Escuela 1 & 52 & 112 & 164 \\
\hline Escuela 2 & 40 & 20 & 60 \\
\hline Escuela 3 & 15 & 64 & 79 \\
\hline Total & 107 & 196 & 303 \\
\hline
\end{tabular}

Tabla 1. Número de participantes del estudio 


\begin{tabular}{|c|c|c|c|c|}
\hline & I Semestre & VII Semestre & Total \\
\hline \multirow[t]{3}{*}{ Sexo femenino } & Escuela 1 & 46 & 104 & 150 \\
\hline & Escuela 2 & 19 & 7 & 26 \\
\hline & Escuela 3 & 12 & 47 & 59 \\
\hline \multicolumn{2}{|l|}{ Subtotal } & 77 & 158 & 235 \\
\hline \multirow[t]{3}{*}{ Sexo masculino } & Escuela 1 & 6 & 8 & 14 \\
\hline & Escuela 2 & 21 & 13 & 34 \\
\hline & Escuela 3 & 3 & 17 & 20 \\
\hline \multicolumn{2}{|l|}{ Subtotal } & 30 & 38 & 68 \\
\hline \multicolumn{2}{|l|}{ Total } & 107 & 196 & 303 \\
\hline
\end{tabular}

Tabla 2. Segmentación de participantes del estudio por sexo y semestre

El proceso de recolección de datos se realizó en una intervención con los grupos de normalistas, en la cual se mencionó el propósito de la investigación. Posteriormente se mostró el instrumento para dar la consigna de la lectura del texto del instrumento, cuya temática es de interés público, con la que responderían el conjunto de ítems para explorar la comprensión del texto. Como parte de las indicaciones para la recolección de datos se señaló que cada participante podría regresar al texto las veces necesarias para responder las preguntas del instrumento, escribir notas, subrayar, etc. como parte de la implementación de estrategias de lectura; todo esto sin límite de tiempo.

El instrumento utilizado tiene un total de 24 ítems, esto permite explorar seis aspectos de la comprensión lectora: 1) identificación de información, 2) nexos entre diversas partes del texto, 3) hipótesis, 4) inferencias, 5) vinculación del texto con situaciones de la vida cotidiana y 6) evaluación crítica. Esta exploración se realiza a través de tres niveles de lectura con los que se utilizan los 24 reactivos. En el primer nivel denominado extracción u obtención de información el lector identifica información presentada en el texto de manera aislada o múltiple. En el segundo nivel, interpretación, el participante elabora el significado y formula alternativas de conclusiones a partir de lo presentado en el texto. Finalmente en el tercer nivel, razonamiento y evaluación, el sustentante relaciona la información del texto con sus conocimientos, ideas y/o experiencias previas (véase tabla 3). Cada nivel de comprensión la lectura se evalúa por ocho reactivos (cuatro de ellos se estudian mediante un indicador y los otros cuatro por otro indicador). Es decir, la suma total de reactivos es de 24 .

\section{Niveles de comprensión \\ Definición \\ Indicadores}




\begin{tabular}{|l|l|l|}
\hline $\begin{array}{l}\text { Nivel 1. Extracción u } \\
\text { obtención de información }\end{array}$ & $\begin{array}{l}\text { Capacidad para localizar } \\
\text { información }\end{array}$ & $\begin{array}{l}1.1 \text { Identificación de } \\
\text { información } \\
1.2 \text { Nexos entre diversas } \\
\text { partes del texto }\end{array}$ \\
\hline $\begin{array}{l}\text { Nivel 2. Interpretación de } \\
\text { textos }\end{array}$ & $\begin{array}{l}\text { Capacidad para construir } \\
\text { significados y hacer } \\
\text { inferencias }\end{array}$ & $\begin{array}{l}2.1 \text { Hipótesis } \\
\text { 2.2 Inferencias }\end{array}$ \\
\hline $\begin{array}{l}\text { Nivel 3. Razonamiento de } \\
\text { contenido }\end{array}$ & $\begin{array}{l}\text { Capacidad para relacionar el } \\
\text { texto con sus conocimientos, } \\
\text { ideas y experiencias }\end{array}$ & $\begin{array}{l}\text { 3.1 Vinculación con la } \\
\text { realidad } \\
\text { 3.2 Evaluación crítica }\end{array}$ \\
\hline
\end{tabular}

Tabla 3. Niveles de comprensión de textos

Finalmente, con el propósito de comprender los datos cuantitativos de una manera cualitativa se utilizó dos escalas. La primera para el análisis de niveles de lectura y la segunda para el total de los resultados obtenidos. Estas escalas se muestran en las tablas 4 y 5, en ambas del lado izquierdo se encuentra el valor cualitativo y del lado derecho el rango de aciertos que obtuvieron los grupos estudiados.

\begin{tabular}{|c|c|}
\hline Logro cualitativo & Logro cuantitativo \\
\hline Excelente & 8 \\
\hline Muy alto & 7 \\
\hline Alto & 6 \\
\hline Medio & $5-3$ \\
\hline Bajo & 2 \\
\hline Muy bajo & 1 \\
\hline Nulo & 0 \\
\hline
\end{tabular}

Tabla 4. Escala utilizada para cada nivel de lectura

\begin{tabular}{|c|c|}
\hline Logro cualitativo & Logro cuantitativo \\
\hline Excelente & 24 \\
\hline Muy alto & $19-23$ \\
\hline Alto & $15-18$ \\
\hline Medio & $10-14$ \\
\hline Bajo & $6-9$ \\
\hline Muy bajo & $1-4$ \\
\hline Nulo & 0 \\
\hline
\end{tabular}

Tabla 5. Escala utilizada para el total de los resultados 


\section{PRUEBAS DE NORMALIDAD}

El instrumento se sometió a pruebas de validez, fiabilidad y normalidad. Algunas las pruebas utilizadas son: Q-Q Normal sin tendencia de aciertos (véase gráfico 1 y 2; véase tabla 6 y 7 ), PP Normal de aciertos (véase gráfico 3 y 4; véase tabla 8 y 9) y curva de normalidad (véase gráfico 5). En estas pruebas de normalidad se observa que el instrumento utilizado tiene las condiciones metodológicas para ser considerado como prueba fiable.

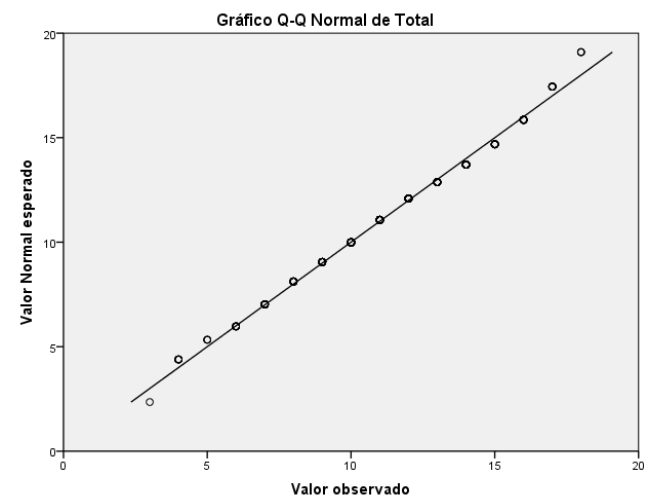

Gráfico 1. Q-Q Normal de total

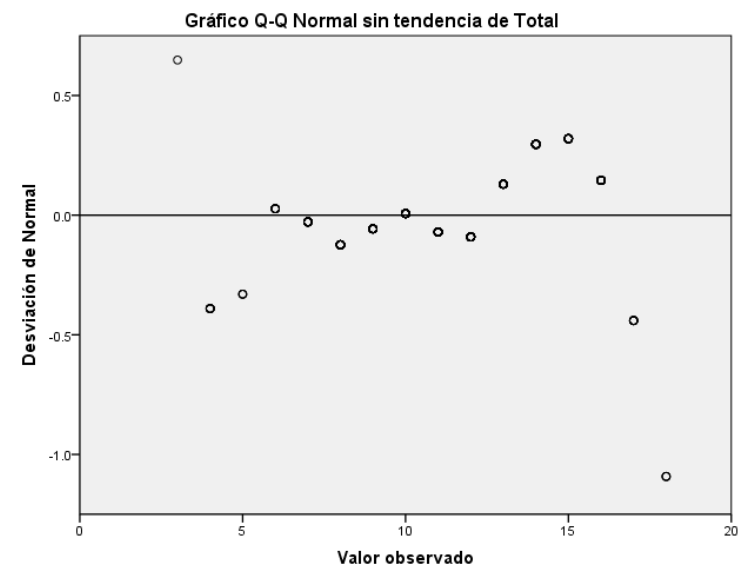

Gráfico 2. Q-Q Normal sin tendencia de total 


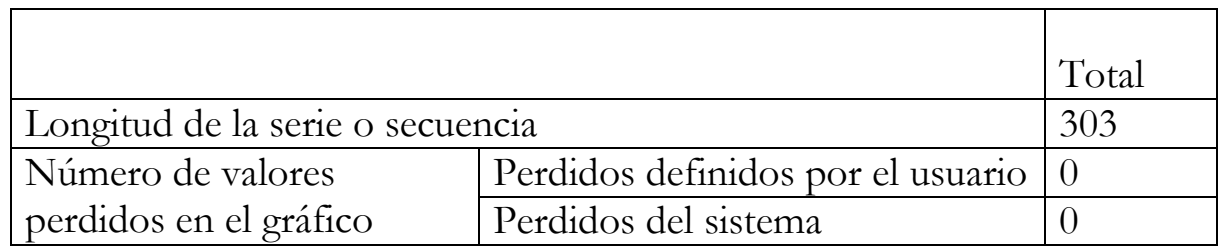

Tabla 6. Resumen del procesamiento de los casos

\begin{tabular}{|l|l|l|}
\hline \multicolumn{2}{|l|}{} & Total \\
\hline \multirow{2}{*}{ Distribución normal } & Ubicación & 11.21 \\
\cline { 2 - 3 } & Escala & 3.088 \\
\hline
\end{tabular}

Tabla 7. Parámetros de distribución estimados

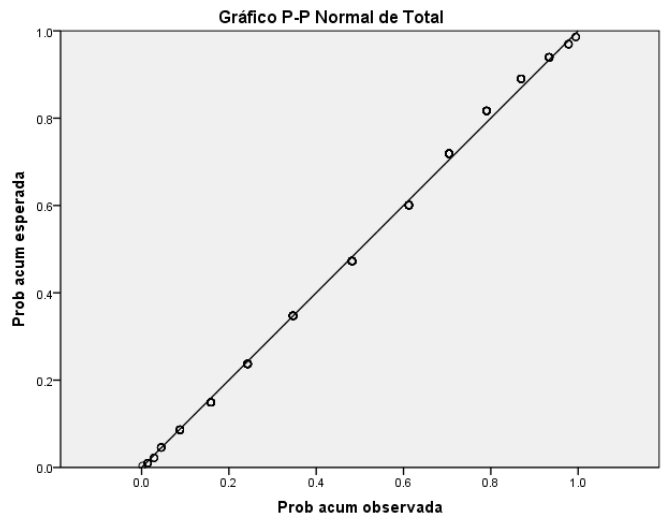

Gráfico 3. P-P Normal de total

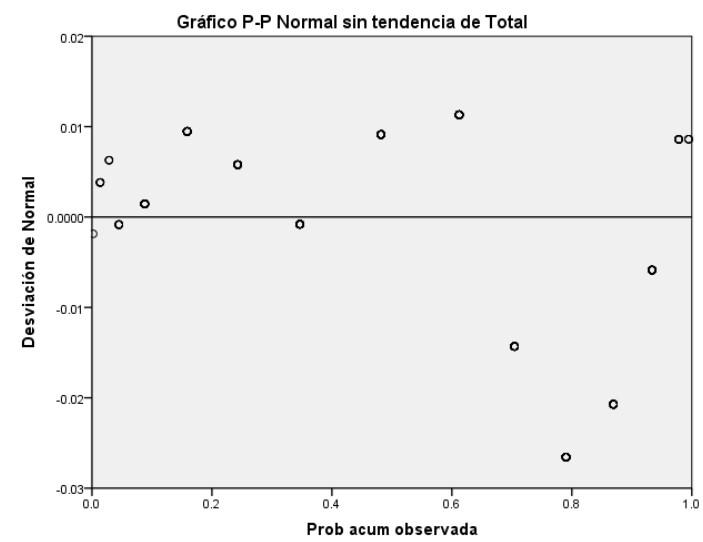

Gráfico 4. P-P Normal sin tendencia de total 


\begin{tabular}{|l|l|l|}
\hline \multicolumn{2}{|l|}{} & Total \\
\hline \multicolumn{2}{|l|}{ Longitud de la serie o secuencia } & 303 \\
\hline $\begin{array}{l}\text { Número de valores } \\
\text { perdidos en el gráfico }\end{array}$ & $\begin{array}{l}\text { Perdidos definidos por } \\
\text { el usuario }\end{array}$ & 0 \\
\cline { 2 - 3 } & Perdidos del sistema & 0 \\
\hline
\end{tabular}

Tabla 8. Resumen del procesamiento de los casos

\begin{tabular}{|l|l|l|}
\hline \multicolumn{2}{|l|}{} & Total \\
\hline Distribución normal & Ubicación & 11.21 \\
\cline { 2 - 3 } & Escala & 3.088 \\
\hline
\end{tabular}

Tabla 9. Parámetros de distribución estimados

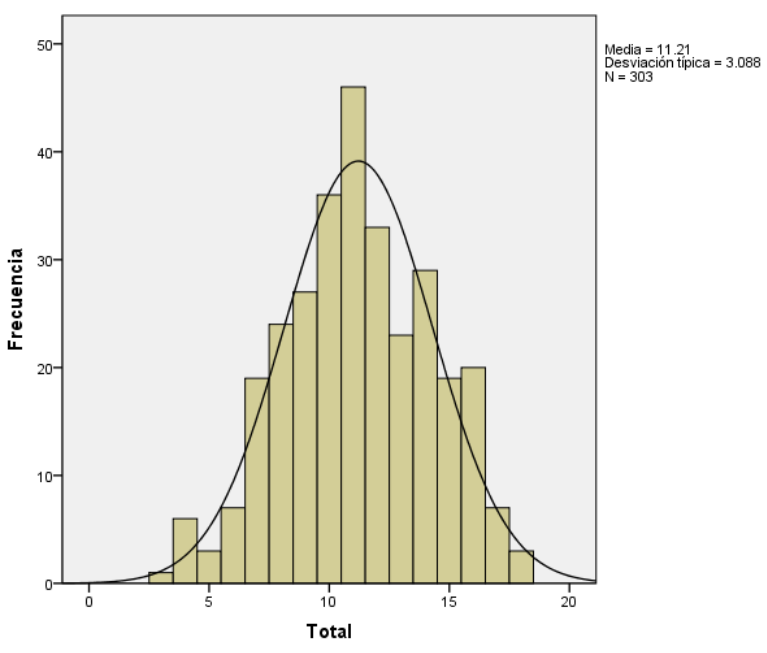

Gráfico 5. Curva de normalidad

Finalmente, se utilizó la prueba Kolmogorov-Smirnov para el tratamiento de la normalidad del instrumento de la presente investigación. En este proceso metodológico se estableció como hipótesis las siguientes enunciaciones: $\mathrm{H}_{0}$ : los datos provienen de una distribución normal (elección al azar) y $\mathrm{H}_{1}$ : los datos no provienen de una distribución normal (elección al azar). Cabe señalar que la elección de esta prueba de normalidad se deriva de la característica de la muestra, la cual es mayor a 30.

\begin{tabular}{|l|l|l|l|}
\hline & Estadístico & gl & Sig. \\
\hline Escuela 1 & .147 & 301 & .000 \\
\hline Escuela 2 & .128 & 301 & .000 \\
\hline Escuela 3 & .130 & 301 & .000 \\
\hline
\end{tabular}

Tabla 10. Prueba de normalidad Kolmogorov-Smirnov 


\section{RESULTADOS}

\section{NIVEL UNO: EXTRACCIÓN U OBTENCIÓN DE INFORMACIÓN}

De acuerdo a las escalas cuantitativas y cualitativas utilizadas en esta investigación, se observa que el logro de los normalistas en el nivel uno, extracción u obtención de información, se sitúa en un rango medio con acercamiento a bajo en algunos de los casos (véase tabla 4, 11 y 12). Asimismo, los datos de las Escuela 2 y Escuela 3 evidencian que para los alumnos de séptimo semestre resultó ligeramente más complejo el nivel uno que para los normalistas de primer semestre, ya que las medias de los estudiantes de séptimo semestre de estas dos escuelas corresponden a 3.95 y 3.87. En cambio, para los alumnos de séptimo semestre de la Escuela 1 fue más sencillo el nivel uno que para los sustentantes de primer semestre de esta escuela, puesto que las medias de ambos semestres son 3.51 y 3.42 respectivamente (véase tabla 11 y 12).

\begin{tabular}{|l|l|l|c|c|}
\hline \multicolumn{2}{|c|}{} & $\begin{array}{c}\text { Extracción u } \\
\text { obtención de } \\
\text { información }\end{array}$ & $\begin{array}{c}\text { Identificación } \\
\text { de } \\
\text { información }\end{array}$ & $\begin{array}{c}\text { Nexos entre } \\
\text { diversas } \\
\text { partes del } \\
\text { texto }\end{array}$ \\
\hline \multirow{2}{*}{ Escuela 1 } & Media & 3.42 & 2.02 & 1.40 \\
\cline { 2 - 5 } & $\mathrm{N}$ & 52 & 52 & 52 \\
\cline { 2 - 5 } & Desv. típ. & 1.109 & 1.000 & .934 \\
\hline Escuela 2 & Media & 3.95 & 2.07 & 1.87 \\
\cline { 2 - 5 } & $\mathrm{N}$ & 40 & 40 & 40 \\
\cline { 2 - 5 } & Desv. típ. & 1.449 & .944 & 1.042 \\
\hline \multirow{3}{*}{ Escuela 3 } & Media & 3.87 & 2.40 & 1.47 \\
\cline { 2 - 5 } & $\mathrm{N}$ & 15 & 15 & 15 \\
\cline { 2 - 5 } & Desv. típ. & .990 & .910 & .915 \\
\hline
\end{tabular}

Tabla 11. Resultados de primer semestre - Nivel 1 


\begin{tabular}{|l|l|c|c|c|}
\hline \multicolumn{2}{|c|}{} & $\begin{array}{c}\text { Extracción u } \\
\text { obtención de } \\
\text { información }\end{array}$ & $\begin{array}{c}\text { Identificación } \\
\text { de } \\
\text { información }\end{array}$ & $\begin{array}{c}\text { Nexos entre } \\
\text { diversas } \\
\text { partes del } \\
\text { texto }\end{array}$ \\
\hline Escuela 1 & Media & 3.51 & 1.77 & 1.74 \\
\cline { 2 - 5 } & $\mathrm{N}$ & 112 & 112 & 112 \\
\cline { 2 - 5 } & Desv. típ. & 1.414 & .870 & .947 \\
\hline Escuela 2 & Media & 3.50 & 1.95 & 1.55 \\
\cline { 2 - 5 } & N & 20 & 20 & 20 \\
\cline { 2 - 5 } & Desv. típ. & 1.357 & .759 & .999 \\
\hline \multirow{3}{*}{ Escuela 3 } & Media & 3.25 & 1.67 & 1.58 \\
\cline { 2 - 5 } & $\mathrm{N}$ & 64 & 64 & 64 \\
\cline { 2 - 5 } & Desv. típ. & 1.480 & .909 & .989 \\
\hline
\end{tabular}

Tabla 12. Resultados de séptimo semestre - Nivel 1

Tanto para los sustentantes de primer como para los de séptimo semestre de las tres escuelas, la vinculación de información entre diferentes partes del texto resultó compleja, situación contraria con la identificación de información expuesta por el autor de la lectura (véase tabla 11 y tabla 12). Aunque los resultados de primer y séptimo semestre de las tres escuelas normales permiten ubicarlos en un rango de logro medio, estos datos muestran que en el séptimo semestre resultaron más complejas aquellas actividades que implican el uso de sus competencias lectoras para obtener información del texto que para los normalistas de primer semestre. Este panorama resulta interesante, puesto que se esperaría que las experiencias académicas y lectoras con las que se forma el normalista promueven el desarrollo de las competencias lectoras y que de alguna u otra manera se reflejaría en este estudio.

\section{NIVEL DOS: INTERPRETACIÓN DE TEXTOS}

A partir de los resultados cuantitativos, los datos indican que los normalistas de primer y séptimo semestre se ubican en el nivel dos, interacción de textos, en un logro cualitativo medio. Sin embargo, los resultados tienen aproximaciones de logro bajo, particularmente con aquellos obtenidos de los estudiantes de primer semestre de la Escuela 1 y Escuela 3 (véase tabla 4, 13 y 14). En el nivel dos se observa que los estudiantes de séptimo semestre lograron mayores resultados que los de primer semestre, puesto que los datos de la Escuela 1, la Escuela 2 y Escuela 3 tienen una media de 4.63, 4.55 y 4.53 respectivamente en comparación de 3.96, 4.53 y 3.73. Asimismo, para los normalistas de séptimo fue más sencillo realizar hipótesis que inferencias; en Noriega doi: https://doi.org/10.36799/el.v4i1.78Volumen 4, Número 1, Año 2019, ISSN: 2448-5942 
cambio para los alumnos de primer semestre de la Escuela 1 y la Escuela 3 fue complejo realizar ambos aspectos del nivel dos (véase tabla 13 y 14).

\begin{tabular}{|l|l|l|l|l|}
\hline \multicolumn{2}{|c|}{} & $\begin{array}{l}\text { Interpretación } \\
\text { de textos }\end{array}$ & Hipótesis & Inferencias \\
\hline \multirow{2}{*}{ Escuela 1 } & Media & 3.96 & 1.98 & 1.98 \\
\cline { 2 - 5 } & $\mathrm{N}$ & 52 & 52 & 52 \\
\cline { 2 - 5 } & Desv. típ. & 1.584 & .874 & 1.075 \\
\hline \multirow{2}{*}{ Escuela 2 } & Media & 4.53 & 2.33 & 2.20 \\
\cline { 2 - 5 } & $\mathrm{N}$ & 40 & 40 & 40 \\
\cline { 2 - 5 } & Desv. típ. & 1.694 & .971 & .992 \\
\hline \multirow{2}{*}{ Escuela 3 } & Media & 3.73 & 1.87 & 1.87 \\
\cline { 2 - 5 } & $\mathrm{N}$ & 15 & 15 & 15 \\
\cline { 2 - 5 } & Desv. típ. & 1.486 & 1.060 & 1.060 \\
\hline
\end{tabular}

Tabla 13. Resultados de primer semestre - Nivel 2

\begin{tabular}{|l|l|c|c|c|}
\hline \multicolumn{2}{|c|}{} & $\begin{array}{c}\text { Interpretación } \\
\text { de textos }\end{array}$ & Hipótesis & Inferencias \\
\hline \multirow{3}{*}{ Escuela 1} & Media & 4.63 & 2.48 & 2.14 \\
\cline { 2 - 5 } & $\mathrm{N}$ & 112 & 112 & 112 \\
\cline { 2 - 5 } & Desv. típ. & 1.376 & 1.022 & .948 \\
\hline \multirow{2}{*}{ Escuela 2 } & Media & 4.55 & 2.50 & 2.05 \\
\cline { 2 - 5 } & $\mathrm{N}$ & 20 & 20 & 20 \\
\cline { 2 - 5 } & Desv. típ. & 2.188 & .889 & 1.504 \\
\hline Escuela 3 & Media & 4.53 & 2.34 & 2.22 \\
\cline { 2 - 5 } & $\mathrm{N}$ & 64 & 64 & 64 \\
\cline { 2 - 5 } & Desv. típ. & 1.671 & .946 & 1.188 \\
\hline
\end{tabular}

Tabla 14. Resultados de séptimo semestre - Nivel 2

La principal característica de estos resultados, a diferencia de los otros niveles, en las tres escuelas se observa un ligero avance en resultados de séptimo semestre en comparación a los de primer semestre. Probablemente, estos resultados son parte de la formación del normalista que de alguna u otra manera estimula la formulación de hipótesis, inferencias e interpretación de diferentes hechos y objetos. Sin embargo, el logro de este nivel de los participantes es medio.

\section{NIVEL TRES: RAZONAMIENTO DE CONTENIDO}

Los datos de primer semestre de la Escuela 1 y Escuela 3, así como los del grupo de séptimo semestre de la Escuela 2 se sitúan en un rango de logro bajo en el nivel tres, Noriega doi: https://doi.org/10.36799/el.v4i1.78Volumen 4, Número 1, Año 2019, ISSN: 2448-5942 
razonamiento de contenido. Respecto a los resultados de los alumnos de séptimo semestre de la Escuela 1 y Escuela 3 y los normalistas de primer semestre de la Escuela 2 se ubican en un rango de logro mediano con aproximación a bajo (véase tabla 4, 15 y 16).

La información obtenida del nivel tres, razonamiento de contenido, muestra la existencia ligera de mayor logro en las medias de los estudiantes de séptimo semestre de la Escuela 1 con 3.61 y la Escuela 3 con 3.55; situación contraria con los normalistas de la Escuela 2, en la que los alumnos de primer semestre alcanzaron 3.18 de media frente a los de séptimo semestre con 2.90. Asimismo, tanto para los alumnos de primero como de séptimo semestre de las tres escuelas fue complicado realizar actividades de evaluación crítica a partir del texto base (véase tabla 15 y 16 ).

\begin{tabular}{|l|l|c|c|c|}
\hline \multicolumn{2}{|c|}{} & $\begin{array}{c}\text { Razonamiento } \\
\text { de contenido }\end{array}$ & $\begin{array}{c}\text { Vinculación } \\
\text { con la } \\
\text { realidad }\end{array}$ & $\begin{array}{c}\text { Evaluación } \\
\text { crítica }\end{array}$ \\
\hline \multirow{2}{*}{ Escuela 1 } & Media & 2.65 & 1.17 & 1.48 \\
\cline { 2 - 5 } & $\mathrm{N}$ & 52 & 52 & 52 \\
\cline { 2 - 5 } & Desv. típ. & 1.297 & .923 & .852 \\
\hline \multirow{2}{*}{ Escuela 2 } & Media & 3.18 & 1.25 & 1.95 \\
\cline { 2 - 5 } & $\mathrm{N}$ & 40 & 40 & 40 \\
\cline { 2 - 5 } & Desv. típ. & 1.615 & 1.080 & 1.011 \\
\hline \multirow{2}{*}{ Escuela 3 } & Media & 2.33 & 1.00 & 1.33 \\
\cline { 2 - 5 } & $\mathrm{N}$ & 15 & 15 & 15 \\
\cline { 2 - 5 } & Desv. típ. & 1.496 & .655 & 1.291 \\
\hline
\end{tabular}

Tabla 15. Resultados de primer semestre - Nivel 3

\begin{tabular}{|l|l|l|c|c|}
\hline \multicolumn{2}{|c|}{} & $\begin{array}{c}\text { Razonamiento } \\
\text { de contenido }\end{array}$ & $\begin{array}{c}\text { Vinculación } \\
\text { con la } \\
\text { realidad }\end{array}$ & $\begin{array}{c}\text { Evaluación } \\
\text { crítica }\end{array}$ \\
\hline \multirow{2}{*}{ Escuela 1 } & Media & 3.61 & 1.67 & 1.94 \\
\cline { 2 - 5 } & $\mathrm{N}$ & 112 & 112 & 112 \\
\cline { 2 - 5 } & Desv. típ. & 1.371 & 1.142 & 1.034 \\
\hline Escuela 2 & Media & 2.90 & 1.05 & 1.85 \\
\cline { 2 - 5 } & $\mathrm{N}$ & 20 & 20 & 20 \\
\cline { 2 - 5 } & Desv. típ. & 1.518 & .945 & .875 \\
\hline Escuela 3 & Media & 3.55 & 1.53 & 2.02 \\
\cline { 2 - 5 } & $\mathrm{N}$ & 64 & 64 & 64 \\
\cline { 2 - 5 } & Desv. típ. & 1.553 & .942 & 1.046 \\
\hline
\end{tabular}

Tabla 16. Resultados de séptimo semestre - Nivel 3 


\section{RESULTADOS GENERALES}

Los resultados cuantitativos del primer semestre de la Escuela 1 y la Escuela 2 corresponden a medias de 10.04 y 11.68 respectivamente, con lo cual al situarlas en la escala de conversión de datos cuantitativos a cualitativos se ubican en un rango medio de comprensión de textos. En el caso de los normalistas de primer semestre de la Escuela 3 obtuvieron 9.93 de media, situándose en un logro bajo de comprensión (véase tabla 5 y tabla 17). Por otra parte, los estudiantes de séptimo semestre de la Escuela 1, la Escuela 2 y la Escuela 3 lograron un rango de comprensión medio, esto a partir de las medias $11.74,10.95$ y 11.33 respectivamente (véase tabla 5 y tabla 18).

\begin{tabular}{|l|r|r|c|}
\hline & \multicolumn{1}{|c|}{ Media } & \multicolumn{1}{c|}{$\mathrm{N}$} & Desv. típ. \\
\hline Escuela 1 & 10.04 & 52 & 2.686 \\
\hline Escuela 2 & 11.68 & 40 & 3.308 \\
\hline Escuela 3 & 9.93 & 15 & 2.492 \\
\hline Total & 10.64 & 107 & 2.995 \\
\hline
\end{tabular}

Tabla 17. Resultados de primer semestre - Total

\begin{tabular}{|l|r|r|c|}
\hline & \multicolumn{1}{|c|}{ Media } & \multicolumn{1}{c|}{$\mathrm{N}$} & Desv. típ. \\
\hline Escuela 1 & 11.74 & 112 & 2.949 \\
\hline Escuela 2 & 10.95 & 20 & 3.720 \\
\hline Escuela 3 & 11.33 & 64 & 3.168 \\
\hline Total & 11.53 & 196 & 3.101 \\
\hline
\end{tabular}

Tabla 18. Resultados de séptimo semestre - Total

En general, los datos de esta investigación permiten ubicar a los participantes en el rango medio de comprensión de textos con una media de 11.21 con una desviación típica de 3.088 (véase tabla 19).

\begin{tabular}{|l|r|r|c|}
\hline & \multicolumn{1}{|c|}{ Media } & \multicolumn{1}{c|}{$\mathrm{N}$} & Desv. típ. \\
\hline Escuela 1 & 11.20 & 164 & 2.969 \\
\hline Escuela 2 & 11.43 & 60 & 3.436 \\
\hline Escuela 3 & 11.06 & 79 & 3.086 \\
\hline Total & 11.21 & 303 & 3.088 \\
\hline
\end{tabular}

Tabla 19. Resultados de tres escuelas - Total 


\section{DISCUSIÓN}

En México en las últimas décadas se han realizado diversas evaluaciones e investigaciones sobre las competencias lectoras de estudiantes de educación básica y media superior, en las cuales los resultados muestran que los mexicanos han logrado niveles bajo y medio-bajo. En la producción de investigación se observa que gran parte de la atención se focaliza en la comprensión de textos de niños y jóvenes que se encuentran dentro del sistema educativo (Arriaga, 2011; Backhoff, Andrade, Peon, Sánchez y Bouzas, 2006; Catalá, Catalá, Molina y Monclús, 2001; Condermarín y Medina, 2000; Díaz, Flores y Martínez, 2007; Fernández y Carlino, 2009; Fuentes, 2009; Gómez, 2008; Vega, Bañales, Reyna y Pérez, 2014). Sin embargo, existe poca producción de investigaciones en las se exploren estas competencias de los futuros profesores de educación primaria y secundaria. La indagación de dichas competencias de alumnos normalistas es necesaria, pues nadie enseña sin saber y tener las habilidades que se busca lograr en los procesos de aprendizajes de otros. En este sentido, el desarrollo de las competencias lectoras de los niños y jóvenes dentro del contexto áulico partirán de aquello que promueva el docente dentro de este espacio, teniendo como base principal las experiencias, las prácticas y las competencias lectoras de los docentes.

La presente investigación tuvo como hipótesis principal que los normalistas de séptimo semestre mostrarían un logro mayor que los de primer semestre, esto considerando las experiencias lectoras y posibles prácticas de lectura que pudieran tener a partir del avance formativo en el que se encuentran. Sin embargo, los datos obtenidos muestran que los participantes logran un rango medio de comprensión lectora y la nula diferencia significativa entre los normalistas de primer y séptimo semestre de las tres escuelas. El instrumento utilizado para la obtención de esta información se caracteriza por ser una prueba objetiva con la que permite tener una aproximación al objeto de estudio. En este sentido se advierte la importancia de la realización de más investigaciones con las que se complemente y aporte al entendimiento del complejo objeto de estudio.

La investigación de este texto muestra la necesidad de crear estrategias de acompañamiento y de formación de lectores para los futuros profesores sonorenses, pues a través de las experiencias y competencias lectoras formarán a los próximos lectores de la sociedad. Probablemente, parte de las bases teóricas de estas estrategias de intervención requerirán ser los modelos de lecturas vinculadas con actividades que generen experiencias 
lectoras que fortalezcan la identificación de información, nexos entre diversas partes del texto, planteamiento de hipótesis, formulación de inferencias, vinculación de los textos con situaciones de la sociedad y evaluación crítica a partir de las lecturas de diversos textos. A manera de propuesta de intervención, se recomienda el diseño y la implementación de asignaturas que tengan por objetivo el desarrollo de las competencias lectoras a partir de la diversidad de la tipología textual, esto en las asignaturas complementarias incluidas en el programa curricular normalista.

\section{CONCLUSIÓN}

Los datos de este estudio ofrecen un acercamiento a las competencias lectoras de los futuros profesores que se están formando en uno de los Estados de la región noroeste de México, y con ello otra perspectiva de las habilidades lectoras de los mexicanos. Los resultados de la investigación muestran que los normalistas de primer semestre obtuvieron 10.64 de media, puntaje que ubica el logro de sus competencias lectoras en un rango medio (véase tabla 5 y 17); en el caso de los participantes de séptimo semestre su media es de 11.21, con este resultado se ubican dentro de la escala de logro en el rango medio (véase tabla 5 y 18). De acuerdo con las hipótesis planteadas, $\mathrm{H}_{0}$ : los datos provienen de una distribución normal (elección al azar) y $\mathrm{H}_{1}$ : los datos no provienen de una distribución normal (elección al azar), con lo cual se concluye que no se presenta una diferencia significativa entre las competencias lectoras de primer y séptimo semestre de las tres escuelas normales. Esto a partir del uso de un $\alpha=0.05$ y el $\mathrm{p}_{\mathrm{v}}$ (significancia asintótica) en Escuela 1, Escuela 2 y Escuela 3 son menor en cada caso que 0.05 se acepta $H_{1}$, aceptando que los datos no provienen de una distribución normal. Lo anterior permite afirmar que de alguna u otra manera, las experiencias y las prácticas lectoras son poco significativas dentro de su proceso de formación académica. Si bien es cierto, la formación académica utiliza textos especializados. No obstante, esta variedad de textos fortalece las experiencias y las prácticas de lectura. Cabe señalar que el tema central del texto utilizado en el instrumento es de interés público, con ello se pretendió que variables como la formación académica, el tipo de texto y el lenguaje del texto no influyeran de manera significativa en los logros de las competencias lectoras de los normalistas.

Los datos evidencian que las competencias lectoras exploradas de los normalistas en el nivel uno extracción u obtención de información y en el nivel dos interpretación de textos se encuentran en un rango medio dentro de la escala de logro. Se observa en los resultados que dos grupos de los 
estudiantes de primer semestre se sitúan en un rango bajo en el nivel tres razonamiento del contenido, y en el caso de los normalistas de séptimo semestre dos grupos se encuentran en un nivel medio. Aunque se observa un avance en el rango con los estudiantes de séptimo semestre, los datos no tienen diferencia significativa.

A partir de lo anteriormente señalado, se afirma que el logro de competencias lectoras de los normalistas participantes de este estudio se encuentra en un rango medio. Se observa la necesidad de crear espacios y herramientas con las que los futuros profesores desarrollen significativamente sus competencias lectoras, no únicamente como herramienta de aprendizaje, sino de interacción dentro de las comunidades escritas y como agentes promotores de competencias lectoras de otros integrantes de estas comunidades.

\section{REFERENCIAS BIBLIOGRÁFICAS}

Aguirre de Quevedo, Lidia; Laura Mandatori de Villagra; Diana Ovejero y Ilda Rojas. "Estudio preliminar sobre predicción y dificultades durante la actividad lectora en alumnos del Departamento Fray M. Esquiú, Catamarca”. Congreso Regional de Ciencia y ecnología NOA. Secretaría de Ciencia y Tecnología, Universidad Nacional de Catamarca, 2002. 20 de febrero 2018. doi: http://www.academia.edu/1343547/

Alonso, Jesús y María del Mar Mateos. “Comprensión lectora: modelos, entrenamiento y evaluación”. Infancia y Aprendizaje, 31-32 (1985): 5-19. 20 de febrero 2018. doi: https://dialnet.unirioja.es/descarga/articulo/667401.pdf

Arriaga Méndez, Juana. "El desarrollo de la comprensión lectora a través de nuevos entornos de lectura". Mundialización educativa, ECE Digital, 3 (2011): 68-84. 16 de enero 2018. doi: http:/ /ece.edu.mx/ecedigital/files/numero3.pdf.

Backhoff Escudero Eduardo, Edgar Andrade Muñoz, Margarita Peon Zapata, Andrés Sánchez Moguel y Arturo Bouzas Riaño. El aprendizaje del Español, las Matemáticas y la Expresión Escrita en Educación Básica en México: sexto de primaria y tercero de secundaria. México: Instituto Nacional para la Evaluación de la Educación, 2006.

Bruning, Roger H.; Schraw, Gregory J.; Norby, M. y Ronning, Royce. Psicología cognitiva y de la instrucción. Madrid: Pearson, 2007. 
Calderón-Ibáñez, Arlenys y Jorge Quijano-Peñuela. “Caracterízticas de comprensión lectora en estudiantes universitarios”. Revista Estudios Socio-Jurídicos, 12.1 (2010): 337-364.

Carlino, Paula y Silvia Martínez (coord.). La lectura y la escritura: un asunto de todos / as. Argentina: Editorial de la Universidad Nacional de Comahue, 2009.

Cassany, Daniel. Prácticas letradas contemporáneas. México: Ríos de tinta, 2008.

Catalá, Gloria, Mireia Catalá, Escarna Molina y Rosa Monclús. Evaluación de la comprensión lectora. Pruebas ACL $\left(1^{\circ}\right.$ - $6^{\circ}$ de primaria). Barcelona: Grao, 2001.

Condemarín Mabel y Alejandra Medina. Programa P-900. Evaluación de los Aprendizajes. Un Medio para Mejorar las Competencias Lingüisticas y Comunicativas. Chile: Ministerio de Educación, 2000.

Del Valle, María José. Variables que inciden en la adquisicón de hábitos de lectura de los estudiantes. Guatemala: Ministerio de Educación, 2012.

Díaz María Antonieta, Gustavo Flores y Felipe Martínez. PIS A 2006 en México. México: Instituto Nacional para la Evaluación de la Educación Superior, 2007.

Dubois, María Eugenia. "Las teorías sobre lectura y la educación superior". Revista Lecturay Vida, 3 (1989): 5-7. 20 de febrero 2018. doi: http://www.lecturayvida.fahce.unlp.edu.ar/numeros/a10n3/10_03_Dubois.pdf/view

Durango Herazo, Zarina Rosa. "Niveles de comprensión lectora en los estudiantes de la Corporación Universitaria Rafael Núñez (Cartagena de Indias)”. Revista Virtual Universidad Cátolica del Norte, 51 (2017): 156-174. 13 de septiembre de 2018. doi: https://www.redalyc.org/pdf/1942/194252398011.pdf

Escorcia, Dyanne. “Conocimientos metacognitivos y autorregulación: una lectura cualitativa del funcionamiento de los estudiantes universitarios en la producción de textos". Avances en Psicología Latinoamericana, 28.2 (2010): 265-277. 25 de febrero 2018. doi: http://www.scielo.org.co/pdf/apl/v28n2/v28n2a10.pdf

Fernández Graciela y Paula Carlino. “¿En qué se diferencian las prácticas de lectura y escritura de la universidad y de la escuela secundaria?". Lectura y Vida, (2009): 6-19. 25 de enero 2018. doi: http://www.lecturayvida.fahce.unlp.edu.ar/numeros/a31n3/31_03_Fernandez.pdf 
Fuentes Monsálves, Liliana. "Diagnóstico de comprensión lectora en educación básica en Villarrica y Loncoche, Chile”. Perfiles educativos, 31.125 (2009): 23-37.

Galera Noguera, Francisco. "La lectoescritura: métodos y procesos”. En Mendoza Fillola, F. (coord.). Didáctica de la lengua y la literatura. Madrid: Prentice Hall, 2003.

Gómez, Luis Felipe. "El desarrollo de la competencia lectora en los primeros grados de primaria". Revista Latinoamericana de Estudios Educativos (México), 38.3-4 (2008): 96-126.

González Casaravilla, Nelson. Comprensión lectora y enfoques de estudio en estudiantes universitarios en un contexto de enseñanza de la redacción de textos académicos y su impacto en el rendimiento académico. Tesis doctoral Universidad de Sonora, Hermosillo, Sonora, 2013.

Goodman, Kenneth. "Reading: A psycholinguistic guessing game". Journal of the Reading. 4 (1967): 126-135.

Goodman, Kenneth. Sobre la lectura. Una mirada de sentido común a la naturaleza del lenguaje y la ciencia de la lectura. México: Paidós, 2006.

Irigoyen, Juan José, Miriam Yerith Jiménez y Karla Fabiola Acuña. “Competencias y educación superior". Revista Mexicana de Investigación Educativa, 16.48 (2011): 243-266.

Molina Villaseñor, Leandro. "Lectura y educación: los hábitos lecotres y su repercusión académica en la Educación Secundaria Obligatoria”. Ocnos: Revista de Estudios sobre Lectura, 2(2006): 103-121.

Monroy Romero, José Alberto y Blanca Estela Gómez López. “Comprensión lectora”. Revista Mexicana de Orientación Educativa, 6.16 (2009): 37-42. 20 de febrero 2018. doi: pepsic.bvsalud.org/pdf/remo/v6n16/v6n16a08.pdf

Ramos Banteurt, Alejandro Arturo y María Nila Blanch Milhet. "Lectura y comprensión. Necesidad de su síntesis entre los estudiantes universitarios". Ciencia en su PC, 3 (2009): 59-66. 14 de mayo del 2018. doi: https://www.redalyc.org/pdf/1813/181321553007.pdf

Rivera Pérez, Santiago Jorge; Maritza Forteza Cáceres y Isabel Cristina Rivera Pérez. "Un modelo teórico sistemático estructural-funcional para el desarrollo de la habilidad de comprensión de lectura". Revista Iberoamericana de Educación, 39.5 (2006). 20 de febrero 2018. doi: http://www.rieoei.org/deloslectores/1461Rivera.pdf

Noriega doi: https://doi.org/10.36799/el.v4i1.78Volumen 4, Número 1, Año 2019, ISSN: 2448-5942 
Seoane, María Nieves. "Lectura e comprensión: una aproximación cognitiva”. Revista Galega do Ensino. 35 (2002):177-198. 20 de febrero 2018. doi: ttps://dialnet.unirioja.es/servlet/articulo?codigo $=2560947$

Smith, Frank. Para darle sentido a la lectura. 2 Edición. Madrid: Visor, 1997.

Solé, Isabel. Estrategia de lectura. 15 Edición. Barcelona: Graó, 2004.

Viglione Elisabeth; María Estela López y María Teresa Zabala. "Implicaciones de diferentes modelos de la ciencia en la comprensión lectora”. Fundamentos en humanidades Universidad Nacional de San Luis. 4.2 (2005) 79-93.

Van Dijk, Teun A., y Walter Kintsch. Strategies of discourse comprehension. Estados Unidos: Academic Press, 1983.

Vega López Norma Alicia, Gerardo Bañales Faz, Antonio Reyna Valladares y Elsa Pérez Amaro Elsa. "Enseñanza de estrategias para la comprensión de textos expositivos con alumnos de sexto grado de primaria”. Revista Mexicana de Investigación Educativa, 19.63 (2014): 10471068.

Villasmil Flores Yeriling, Beatriz Arrieta de Meza y Gloria Fuenmayor de Vílchez. “Análisis de la comprensión lectora y producción escrita de educación media diversificada y profesional". Multiciencias, 9.1 (2009): 62-69.

Zannoto, Mercedes, Carles Monereo y Montserrat Castelló. "Estrategias de lectura y producción de textos académicos. Leer para evaluar un texto científico”. Perfiles Educativos, 33.133 (1001): 10-29. 26 de febrero de 2018. doi: http://www.scielo.org.mx/scielo.php?script=sci_arttext\&pid=S0185-26982011000300002 
